\title{
The antiferromagnetic Ising chain in a mixed transverse and longitudinal magnetic field
}

\author{
A.A.Ovchinnikov, D.V.Dmitriev $*$ and V.Ya.Krivnov \\ Joint Institute of Chemical Physics of RAS, Kosygin str.4, 117977, Moscow, Russia. \\ V.O.Cheranovskii \\ Institute of Chemistry, Kharkov National University, 61077, Kharkov, Ukraine
}

(Dated: February 2, 2008)

\begin{abstract}
We have studied the antiferromagnetic Ising chain in a transverse magnetic field $h_{x}$ and uniform longitudinal field $h_{z}$. Using the density matrix renormalization group calculation combined with a finite-size scaling the ground state phase diagram in $\left(h_{x}, h_{z}\right)$ plane is determined. It is shown that there is an order-disordered transition line in this plane and the critical properties belong to the universality class of the two-dimensional Ising model. Based on the perturbation theory in $h_{z}$ the scaling behavior of the mass gap in the vicinity of the critical point $\left(h_{x}=1 / 2, h_{z}=0\right)$ is established. It is found that the form of the transition line near the classical multicritical point $\left(h_{x}=0, h_{z}=1\right)$ is linear. The connection of the considered quantum model with the quasi-one-dimensional classical Ising model in the magnetic field is discussed.
\end{abstract}

\section{INTRODUCTION}

Recently, the study of the field-induced effects in low-dimensional quantum spin systems has been attracting much interest from theoretical and experimental points of view [1], 2], 3], [4] . For the system with an anisotropy of exchange interactions the magnetic properties essentially depend on the direction of the applied magnetic field. For example, the behavior of the one-dimensional antiferromagnetic $X X Z$ model in a transverse magnetic field is drastically different in comparison with the case of the longitudinal field. In particular, the transverse field induces the staggered magnetization in the perpendicular direction and the continuous phase transition takes places at some critical field $[5],[6],[7],[8]$. This effect has been observed in quasi-one-dimensional antiferromagnet $\mathrm{Cs}_{2} \mathrm{CoCl}_{4}[9]$, where the magnetic field has both the transverse and longitudinal components. Therefore, it is important to study the properties of the antiferromagnetic $s=\frac{1}{2} X X Z$ model in mixed transverse and longitudinal magnetic fields

$$
H=\sum\left(S_{n}^{x} S_{n+1}^{x}+S_{n}^{y} S_{n+1}^{y}+\Delta S_{n}^{z} S_{n+1}^{z}\right)-h_{x} \sum S_{n}^{x}-h_{z} \sum S_{n}^{z}
$$

We consider the most simple case of this model - the antiferromagnetic Ising chain given by the Hamiltonian

$$
H=\sum S_{n}^{z} S_{n+1}^{z}-h_{x} \sum S_{n}^{x}-h_{z} \sum S_{n}^{z}
$$

In spite of the simple form of this Hamiltonian it cannot be solved exactly. In [10] the ground state phase diagram of this model has been investigated using numerical diagonalization of finite systems and finite size scaling procedure. It was found [10] that the transition line between the ordered and disordered phases exists and it was assumed that the model belongs to the universality class of the two-dimensional Ising model.

We note that the ferromagnetic Ising chain in the mixed fields has been studied intensively [11, 12], 13]. Though the ferromagnetic and the antiferromagnetic Ising chains in the transverse magnetic field are equivalent, properties of these two models are very different at $h_{z} \neq 0$. (In fact, the model (2) can be transformed to the ferromagnetic chain but in a staggered longitudinal field.) For example, the ground state phase transition in the ferromagnetic model is smeared out by the longitudinal field in contrast to the antiferromagnetic model for which the phase transition remains at $h_{z} \neq 0$.

In this paper we study the model (2) using the Density Matrix Renormalization Group (DMRG) technique [14]. This method allows us to consider the systems up to a few hundred sites and to determine the transition line with high accuracy. Using the finite-size estimation of the ground state energy and low-lying excitations we will show that the model (2) on the transition line is described by the conformal field theory with the central charge $c=\frac{1}{2}$. Besides, we consider the behavior of the model in the vicinity of the special points $h_{x}=\frac{1}{2}, h_{z}=0$ and $h_{x}=0, h_{z}=1$ and determine the form of the transition line near these points.

The paper is organized as follows. In Sec.II we will provide a qualitative physical picture of the ground state phase diagram based on the classical approach. In Sec.III and Sec.IV the behavior of the system in the vicinity of the special points of the critical line will be considered. In Sec.V we will present the DMRG calculation of the critical 
line. Sec.VI is devoted to the connection of the model (2) with the statistical quasi-one-dimensional Ising model in the external magnetic field. In Summary we discuss our results.

\section{THE CLASSICAL APPROACH}

In order to provide a physical picture of the phase diagram of the model (2) we use the classical approximation, when spins are represented as three-dimensional vectors. The classical ground state is given by a configuration in which all spin vectors lie in the $X Z$ plane with the spins on odd and even sites pointing respectively at angles $\varphi_{1}$ and $-\varphi_{2}$ with respect to the $X$ axis. The classical energy of this state is

$$
E / N=-\frac{1}{4} \sin \varphi_{1} \sin \varphi_{2}-\frac{h_{x}}{4}\left(\cos \varphi_{1}+\cos \varphi_{2}\right)-\frac{h_{z}}{4}\left(\sin \varphi_{1}-\sin \varphi_{2}\right)
$$

The angles $\varphi_{1}$ and $\varphi_{2}$, minimizing this energy are solutions of the following equations

$$
\begin{aligned}
& \cos \varphi_{1} \sin \varphi_{2}+h_{z} \cos \varphi_{1}-h_{x} \sin \varphi_{1}=0 \\
& \sin \varphi_{1} \cos \varphi_{2}-h_{z} \cos \varphi_{2}-h_{x} \sin \varphi_{2}=0
\end{aligned}
$$

The solution of these equations is simple in the particular cases $h_{z}=0$

$$
\begin{aligned}
& \varphi_{1}=\varphi_{2}=\arccos \left(h_{x}\right), \quad h_{x} \leq 1 \\
& \varphi_{1}=\varphi_{2}=0, \quad h_{x}>1
\end{aligned}
$$

and $h_{x}=0$

$$
\begin{array}{ll}
\varphi_{1}=\varphi_{2}=\frac{\pi}{2}, & h_{z} \leq 1 \\
\varphi_{1}=-\varphi_{2}=\frac{\pi}{2}, & h_{z}>1
\end{array}
$$

But in general case, when $h_{x} \neq 0$ and $h_{z} \neq 0$, the phase diagram is divided on two regions (see Fig. 1). In the paramagnetic region (PM) the energy minimum is given by the configuration with $\varphi_{1}=-\varphi_{2}=\varphi$, with $\varphi$ determined by the equation

$$
h_{z} \cos \varphi-h_{x} \sin \varphi=\sin \varphi \cos \varphi
$$

The antiferromagnetic (AF) region is characterized by non-zero staggered magnetizations in both $X$ and $Z$ directions

$$
\begin{aligned}
& S_{2 n}^{x}-S_{2 n+1}^{x}=\sin \left(\frac{\varphi_{2}-\varphi_{1}}{2}\right) \sin \left(\frac{\varphi_{1}+\varphi_{2}}{2}\right) \\
& S_{2 n}^{z}-S_{2 n+1}^{z}=\cos \left(\frac{\varphi_{2}-\varphi_{1}}{2}\right) \sin \left(\frac{\varphi_{1}+\varphi_{2}}{2}\right)
\end{aligned}
$$

with $\varphi_{1}+\varphi_{2} \neq 0$.

Thus, in the AF region the magnetic fields induce a perpendicular antiferromagnetic long-range order (LRO). In the classical approach the value $\left(\varphi_{1}+\varphi_{2}\right)$ plays the role of the LRO and it vanishes on the transition line determined by the equations

$$
\begin{aligned}
& h_{x} \cos \varphi+h_{z} \sin \varphi=1 \\
& h_{z} \cos \varphi-h_{x} \sin \varphi=\sin \varphi \cos \varphi
\end{aligned}
$$

where $\varphi_{1}=-\varphi_{2}=\varphi$.

The solution of Eqs. (9) gives the transition line in the explicit form

$$
h_{z}=\sqrt{1-h_{x}^{2 / 3}}\left(1+h_{x}^{2 / 3}\right)
$$

The classical phase diagram is shown on Fig. 1. The transition (critical) line separates the phase with non-zero AFM LRO from the phase with uniform magnetization (the paramagnetic phase).

Of course, the classical approach does not give the correct description of the phase transition. At first, the quantum fluctuations shift the critical point $h_{x}=1$ to $h_{x}=\frac{1}{2}$ at $h_{z}=0$. Secondly, the form of the critical line is certainly incorrect at $h_{x} \ll 1$. Besides, the order parameter $\left(\varphi_{1}+\varphi_{2}\right)$ vanishes on the critical line with the critical exponent $\frac{1}{2}$, which is not valid at least in the critical point $h_{x}=\frac{1}{2}, h_{z}=0$. Nevertheless, the fact of the generation of the staggered magnetizations perpendicular to the field direction is qualitatively true. 


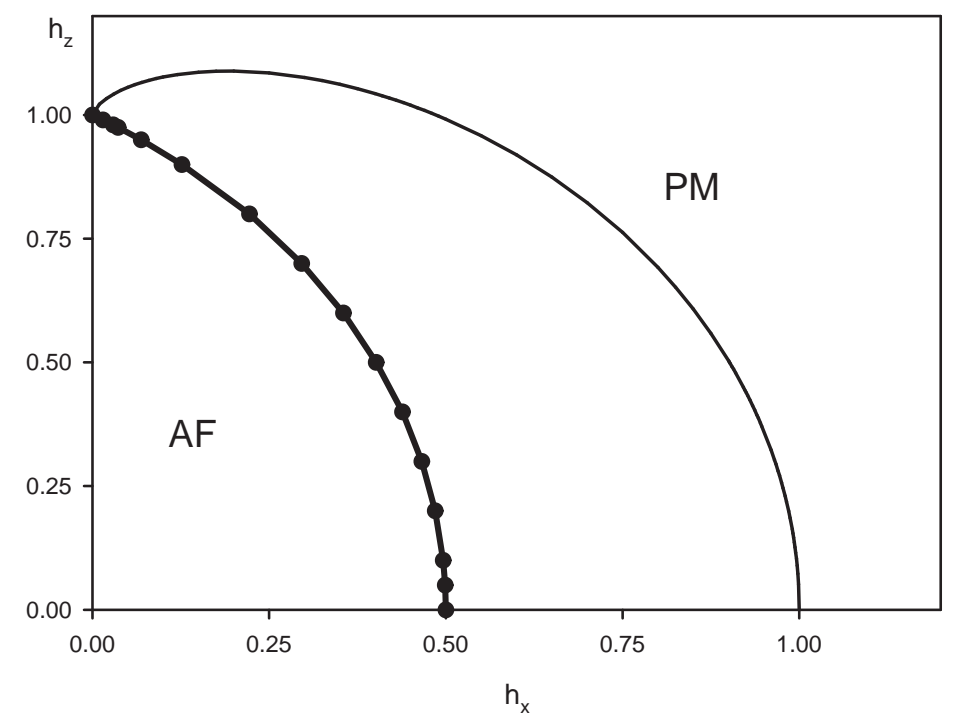

FIG. 1: The ground state phase diagram of the model (2). The critical line between the antiferromagnetic and paramagnetic states obtained from the DMRG calculation is shown by thick solid line and that in the classical approximation by thin solid line.

\section{THE CRITICAL ITF POINT}

Before the numerical determination of the critical line we consider the behavior of the model (2) in the vicinity of two special points: $h_{x}=\frac{1}{2}, h_{z}=0$ and $h_{x}=0, h_{z}=1$. This will give us the form of the critical line near these points.

In the case $h_{z}=0$ the model (2) reduces to the exactly solvable one-dimensional Ising model in the transverse field (the ITF model). This model is well studied [15] and it belongs to the universality class of the two-dimensional Ising model. At $h_{x}<\frac{1}{2}$ the ITF model is gapped and there is non-zero staggered magnetization $\left\langle(-1)^{n} S_{n}^{z}\right\rangle$. The ground state at $h_{x}<\frac{1}{2}$ is two-fold degenerated in the thermodynamic limit. At the point $h_{x}=\frac{1}{2}$ the model becomes gapless and the staggered magnetization vanishes with the critical exponent $\frac{1}{8}$. This is the transition point from the antiferromagnetic state to the paramagnetic gapped state. The gap vanishes linearly at $h_{x}=\frac{1}{2}$.

Now we consider the ITF model with small longitudinal magnetic field. For small magnetic field $h_{z}$ we rewrite the Hamiltonian (2) in the form

$$
\begin{aligned}
H & =H_{0}+V \\
H_{0} & =\sum S_{n}^{z} S_{n+1}^{z}-h_{x} \sum S_{n}^{x} \\
V & =-h_{z} \sum S_{n}^{z}
\end{aligned}
$$

After Jordan-Wigner transformation to the Fermi operators $c_{n}^{+}, c_{n}$

$$
\begin{aligned}
S_{n}^{z} & =\frac{c_{n}^{+}+c_{n}}{2} \prod_{j<n}\left(1-2 c_{j}^{+} c_{j}\right) \\
S_{n}^{x} & =\frac{1}{2}-c_{n}^{+} c_{n}
\end{aligned}
$$

the ITF Hamiltonian $H_{0}$ takes a bilinear form

$$
H_{0}=h_{x} \sum\left(c_{n}^{+} c_{n}-\frac{1}{2}\right)+\frac{1}{4} \sum\left(c_{n}^{+}-c_{n}\right)\left(c_{n+1}^{+}+c_{n+1}\right)
$$

The Hamiltonian $H_{0}$ commutes with the 'parity' operator

$$
P=\exp \left(i \pi \sum c_{n}^{+} c_{n}\right)
$$

because $H_{0}$ can change the number of $c_{n}$ excitation by an even number only. Therefore, the space of states of $H_{0}$ is divided on two sectors with odd $(P=-1)$ and even $(P=1)$ number of the Fermi particles $c_{n}$. 
The Hamiltonian $H_{0}$ is diagonalized exactly [16]

$$
H_{0}=\sum \varepsilon_{k}\left(\eta_{k}^{+} \eta_{k}-\frac{1}{2}\right)
$$

with Fermi particles $\eta_{k}$ and spectrum

$$
\varepsilon_{k}^{2}=h_{x}^{2}+\frac{1}{4}+h_{x} \cos k
$$

which gives a gap at momentum $\pi$

$$
m=\left|h_{x}-\frac{1}{2}\right|
$$

The gap vanishes at the critical point $h_{x}=1 / 2$, where the spectrum becomes

$$
\varepsilon_{k}=\cos \frac{k}{2}
$$

Below in this section we will consider the perturbation theory in $V$ (11) in the critical point $h_{x}=1 / 2$.

The transition operator $S^{z}=\sum S_{n}^{z}$ in $V$ (11) conserves the momentum and changes the 'parity', because as it follows from Eq. (12) it changes the number of the Fermi particles $c_{i}$ (and also the number of $\eta_{k}$ particles) by odd number. Therefore, the non-zero matrix elements with the transition operator $S^{z}$ have the states with equal momentum but different parity. The last fact means that the perturbation theory in $V$ contains only even orders.

The second order correction to the ground state energy is:

$$
\delta E_{0}^{(2)}=h_{z}^{2} \sum_{s} \frac{\left\langle 0\left|S^{z}\right| s\right\rangle\left\langle s\left|S^{z}\right| 0\right\rangle}{E_{0}-E_{s}}
$$

The ground state $|0\rangle$ has the momentum $q=0$ and zero number of the $\eta_{k}$ particles $(P=1)$. Therefore, the non-zero contribution to the sum in (19) is given by the intermediate states $|s\rangle$ with zero momentum and the parity $P=-1$. As follows from Eq.(18), all states $|s\rangle$ with momentum $q=0$ and odd number of the $\eta_{k}$ particles $(P=-1)$ have 'high' energies $E_{s}-E_{0} \equiv \varepsilon_{s} \gtrsim 1$. On the contrary, among the states with $q=0$ and even number of the $\eta_{k}$ particles $(P=1)$ there are many states like $\eta_{\pi-k}^{+} \eta_{-\pi+k}^{+}|0\rangle$ with small $k$ having small excitation energies $\varepsilon_{s} \sim k$ and they can lead to infrared divergencies.

Hereinafter we consider large but finite systems of length $N$. We shall study the dependence of dominant contributions to the perturbation theory on $N$, omitting numerical factors. Using the fact that $\varepsilon_{s} \gtrsim 1$ one can rewrite Eq. (19) as

$$
\delta E_{0}^{(2)} \sim-h_{z}^{2} \sum_{s}\left\langle 0\left|S^{z}\right| s\right\rangle\left\langle s\left|S^{z}\right| 0\right\rangle=-h_{z}^{2}\left\langle 0\left|\left(S^{z}\right)^{2}\right| 0\right\rangle
$$

In higher orders of the perturbation series for the ground state energy each second intermediate state $|s\rangle$ has odd number of the Fermi particles $(P=-1)$, and, therefore, high energy $\varepsilon_{s} \geq 1$. For example, let us consider the fourth order correction to the ground state energy

$$
\begin{aligned}
\delta E_{0}^{(4)}= & h_{z}^{4} \sum_{s, s^{\prime}, s^{\prime \prime}} \frac{\left\langle 0\left|S^{z}\right| s\right\rangle\left\langle s\left|S^{z}\right| s^{\prime}\right\rangle\left\langle s^{\prime}\left|S^{z}\right| s^{\prime \prime}\right\rangle\left\langle s^{\prime \prime}\left|S^{z}\right| 0\right\rangle}{\left(E_{0}-E_{s}\right)\left(E_{0}-E_{s^{\prime}}\right)\left(E_{0}-E_{s^{\prime \prime}}\right)} \\
& -\delta E_{0}^{(2)} h_{z}^{2} \sum_{s} \frac{\left\langle 0\left|S^{z}\right| s\right\rangle\left\langle s\left|S^{z}\right| 0\right\rangle}{\left(E_{0}-E_{s}\right)^{2}}
\end{aligned}
$$

All intermediate states $s, s^{\prime}, s^{\prime \prime}$ have momentum $q=0$. The states $s^{\prime}$ have $P=1$ and some of them have small excitation energies $\varepsilon_{s^{\prime}} \sim 1 / N$, while the states $s$ and $s^{\prime \prime}$ have $P=-1$ and high energies $\varepsilon_{s} \geq 1$. Therefore, one can sum over the intermediate states $s$ and $s^{\prime \prime}$, which reduces Eq.(21) to

$$
\delta E_{0}^{(4)} \sim h_{z}^{4} \sum_{s^{\prime}} \frac{\left\langle 0\left|\left(S^{z}\right)^{2}\right| s^{\prime}\right\rangle\left\langle s^{\prime}\left|\left(S^{z}\right)^{2}\right| 0\right\rangle}{E_{0}-E_{s^{\prime}}}
$$


Now we note, that this expression looks like the second order correction and Eq. (20) looks like the first order correction to the ground state energy with a perturbation $-h_{z}^{2}\left(S^{z}\right)^{2}$. In a similar way one can sum over all intermediate states with $q=0$ and $P=-1$ in all orders of perturbation series. As a result, we arrive at the perturbation theory with the effective perturbation

$$
V_{1}=-h_{z}^{2}\left(S^{z}\right)^{2}=-h_{z}^{2} \sum_{n, m} S_{n}^{z} S_{m}^{z}
$$

We note, that the perturbation theory with the perturbation $V_{1}$ coincides with the original perturbation theory (11) in a sense, that both perturbation series have the same order of divergencies (or power of $N$ ) at each order in $h_{z}$. But numerical factors at each order in $h_{z}$ can be different.

The perturbation $V_{1}$ commutes with the parity operator (14) and conserves the momentum. Therefore, the perturbation series in $V_{1}$ contains the intermediate states with $q=0$ and $P=1$ only, and some of these states have small excitation energies $\varepsilon_{s} \sim 1 / N$. These states give the most divergent contribution to the perturbation series in $V_{1}$ and further we shall take into account these states only.

Now we need to estimate the matrix elements of the operator $\left(S^{z}\right)^{2}$. The behavior of the correlation function $\left\langle S_{n}^{z} S_{m}^{z}\right\rangle$ in the ground state and in the low-lying states with excitation energies $\varepsilon_{s} \sim 1 / N$ on large distances is known [17]

$$
\left\langle S_{n}^{z} S_{m}^{z}\right\rangle \sim \frac{(-1)^{n-m}}{|n-m|^{1 / 4}}
$$

and, therefore, due to oscillation of the correlator $\left\langle S_{n}^{z} S_{m}^{z}\right\rangle$ the sum over $n$ and $m$ can be estimated as

$$
\sum_{n, m}\left\langle S_{n}^{z} S_{m}^{z}\right\rangle=\frac{N}{4}+2 N \sum_{n>1}\left\langle S_{1}^{z} S_{n}^{z}\right\rangle \simeq 0.07465(1) N
$$

where the constant was found from extrapolation of the exact results for finite chains with $N=6, \ldots 14$.

The non-diagonal matrix elements of the operator $\left(S^{z}\right)^{2}$ with two different low-lying states was also calculated numerically. It was found that the only non-zero matrix elements (in the thermodynamic limit) are given by the states $s, s^{\prime}$ differing by two $\eta_{k}$ particles, like $\left|s^{\prime}\right\rangle=\eta_{k}^{+} \eta_{-k}^{+}|s\rangle$. All such pairs of states give the same value for the matrix element

$$
\left\langle s\left|\left(S^{z}\right)^{2}\right| s^{\prime}\right\rangle=0.3108(1)
$$

while all other matrix elements exponentially drop with $N$.

Now we estimate all terms of perturbation series in $h_{z}$. According to Eq.(25) the second order correction (20) is proportional to $h_{z}^{2} N$. The factor at $h_{z}^{2} N$ was found numerically by exact diagonalization of finite systems and the following calculation of the sum (19). The data for $N=6, \ldots 14$ are well extrapolated and give

$$
\delta E_{0}^{(2)}=-0.07060(5) h_{z}^{2} N
$$

According to Eq.(27) the zero-field susceptibility is $\chi_{z}=0.1412(1)$. This result is in a perfect agreement with the value $\chi_{z}=0.14118 \ldots$ obtained analytically in [18].

Using Eq.(26) we find that the non-zero contribution to the fourth order correction to the ground state energy (22) is given by the states $|s\rangle=\eta_{k}^{+} \eta_{-k}^{+}|0\rangle$ with the excitation energy $\varepsilon_{s}=2 \cos \frac{k}{2}$. Thus, because of small excitation energies at $k \sim \pi$ in denominator in (22) the fourth order correction to the ground state energy turns out to be proportional to $N$ (we omit here logarithmic corrections)

$$
\delta E_{0}^{(4)} \sim h_{z}^{4} \sum_{s} \frac{1}{\varepsilon_{s}} \sim h_{z}^{4} N
$$

The $m$-th order in $V_{1}$ is proportional to $h_{z}^{2 m}$. The denominator of the $m$-th order contains $(m-1)$ small excitation energies $\sim 1 / N$ and all matrix elements in the numerator are of the order of unity. Therefore, the $m$-th order in $V_{1}$ diverges as $h_{z}^{2 m} N^{m-1}$. But this is not valid for odd orders in $V_{1}$. The analysis shows that all odd orders in $V_{1}$ diverge as $h_{z}^{2 m} N^{m-2}$. For example, the third order correction in $V_{1}$ (the sixth order in $h_{z}$ ) for the ground state energy does not diverge:

$$
\delta E_{0}^{(6)} \sim h_{z}^{6} N
$$


So, the odd orders in $V_{1}$ give the next order corrections to the ground state energy and we omit it below.

Summarize all above, we arrive at the perturbation series in the form

$$
\delta E_{0}=-a_{0} h_{z}^{2} N-h_{z}^{4} N \sum_{n=0}^{\infty} b_{n}\left(h_{z}^{2} N\right)^{n}
$$

with $a_{0}=0.07059 \ldots$ (Eq. (27) ) and unknown constants $b_{n}$. One can see that the first divergence appears only in the eight-th order in $h_{z}$, and it is very difficult to observe it numerically. The exact numerical calculations up to $N=14$ of the second, the fourth and the sixth order corrections in $h_{z}$ confirm the form of the series (30).

The sum in Eq.(30) forms the scaling function $f_{0}(x)$ of the scaling parameter $x=h_{z}^{2} N$. Thus, the ground state energy takes the form

$$
\delta E_{0}=-a_{0} h_{z}^{2} N-h_{z}^{4} N f_{0}(x)
$$

Since the ground state energy is proportional to $N$, the scaling function $f_{0}(x)$ has finite thermodynamic limit at $x \rightarrow \infty$. Thus, the leading term of the perturbation theory for the ground state energy is given by the second order and the divergent part of the perturbation theory gives the correction $\sim h_{z}^{4}$.

The perturbation series for low-lying states has the same form as in Eq.(31), but each low-lying state has its own scaling function. Therefore, for the first excited state (with momentum $q=\pi$ ) one has

$$
\delta E_{\pi}=-a_{\pi} h_{z}^{2} N-h_{z}^{4} N f_{\pi}(x)
$$

So, the mass gap $m=\delta E_{\pi}-\delta E_{0}$ appears as:

$$
m=a_{m} h_{z}^{2}+g(x) h_{z}^{2}
$$

where $a_{m}=\left(a_{0}-a_{\pi}\right) N$ and the scaling function $g(x)=x\left(f_{0}(x)-f_{\pi}(x)\right)$. Since the gap is finite, the scaling function $g(x)$ in the thermodynamic limit at $x \rightarrow \infty$ must tend to some finite limit

$$
m=a_{m} h_{z}^{2}+g(\infty) h_{z}^{2}
$$

From the last equation we see that the gap is proportional to $h_{z}^{2}$, but the factor at $h_{z}^{2}$ is given not only by the second order correction $a_{m}$ but by all collected divergent orders of the perturbation series. The numerical estimation of the second order correction to the gap gives

$$
a_{m}=0.1875(2)
$$

In order to find the factor at $h_{z}^{2}$ in mass gap we performed DMRG calculations (for details see Sec.V) of the model (11) for $h_{z}=0, \ldots 0.3$ and $N=20, \ldots 300$. The dependence of the gap on the scaling parameter $x=h_{z}^{2} N$ is shown on Fig. 2 One can see that the points with different $N$ and $h_{z}$ lie perfectly on one curve. It definitely manifests that the scaling parameter is $h_{z}^{2} N$. The DMRG calculation data give the mass gap

$$
m=0.37(1) h_{z}^{2}
$$

From this equation follows that the second order correction to the mass gap (35) gives approximately one half of a total gap, and another half is collected by all other divergent orders contained in the scaling function $g(x)$.

We note, that in contrast to Eq. (36) for the ferromagnetic ITF model the mass gap at the critical point $h_{x}=\frac{1}{2}$ is proportional to $h_{z}^{8 / 15}[12],[13]$.

\section{Mapping to the effective model}

Let us return to the estimation of the diagonal element of the operator $V_{1}$ (25). Using the fact of the oscillation of the correlator (24) in the ground state and in the low-lying states we can rewrite the sum in (25) approximately as a one half of the first term:

$$
\left\langle\left(S^{z}\right)^{2}\right\rangle=\frac{N}{4}+2 \sum_{n<m}\left\langle S_{n}^{z} S_{m}^{z}\right\rangle \simeq \frac{N}{4}+\sum_{n}\left\langle S_{n}^{z} S_{n+1}^{z}\right\rangle
$$




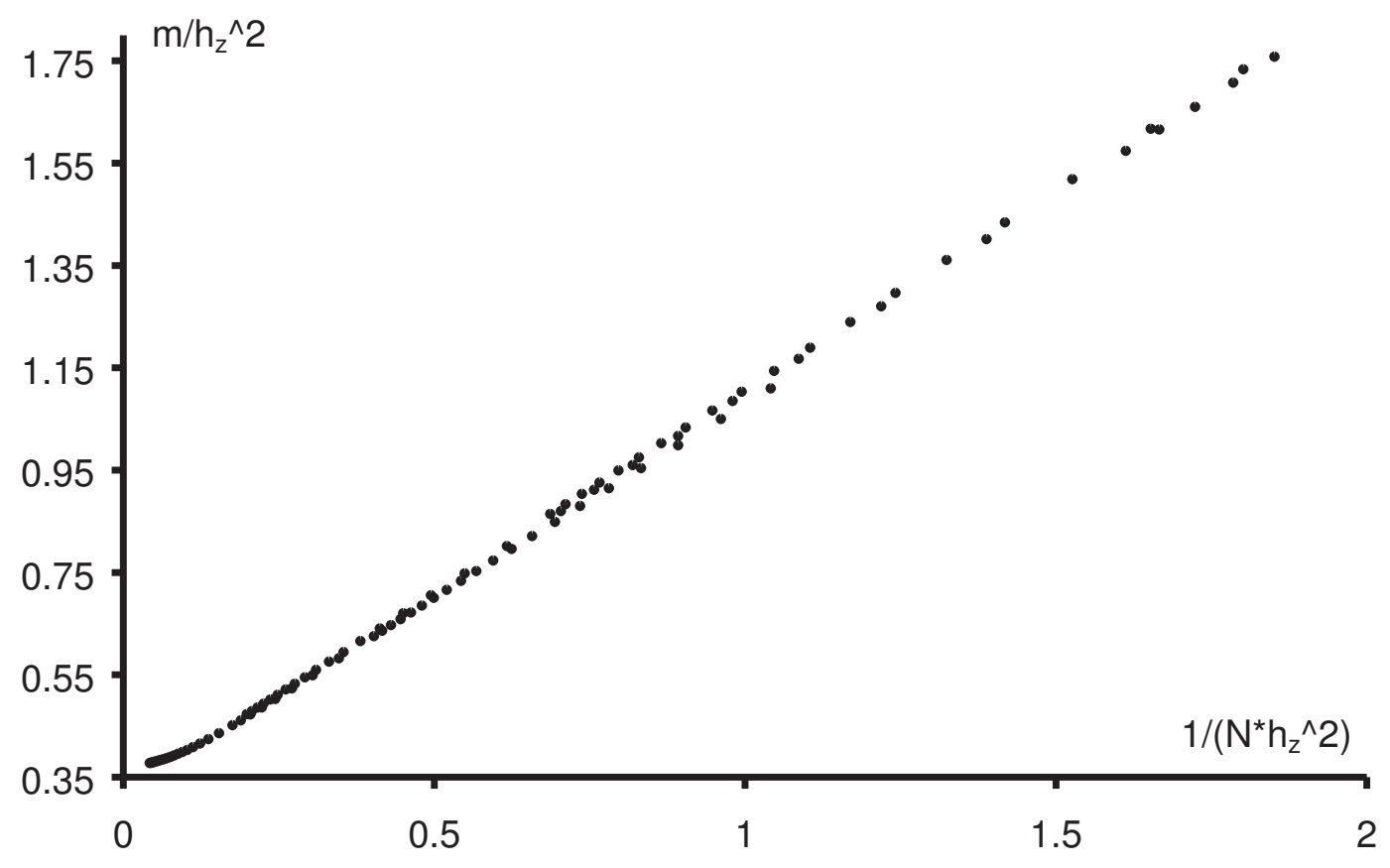

FIG. 2: Scaled mass gap $\frac{m}{h_{z}^{2}}$ for $h_{x}=\frac{1}{2}$ with various values of $N$ and $h_{z}$ as a function of the reciprocal scaled parameter $\frac{1}{N h_{z}^{2}}$.

The last equation suggests that the perturbation $V_{1}$ can be reduced to the operator $V_{2}$

$$
V_{1} \rightarrow V_{2}=-a h_{z}^{2} N-b h_{z}^{2} \sum S_{n}^{z} S_{n+1}^{z}
$$

with some constants $a, b$, which we will define later. In order to verify this assumption we compared the matrix elements of the operator $V_{2}$ with those of $V_{1}$. We have found that the dependence of the matrix elements of the operator $V_{2}$ on $N$ is in a full accord with Eqs.(25) and (26). That is, the diagonal matrix elements of $V_{2}$ are proportional to $N$ and the only non-zero (non-diagonal) matrix elements have the pairs of states differing by two $\eta_{k}$ particles

$$
\left\langle s\left|S_{n}^{z} S_{n+1}^{z}\right| s^{\prime}\right\rangle=\frac{1}{2}
$$

Thus, we arrive at the effective Hamiltonian

$$
H_{\mathrm{eff}}=-a h_{z}^{2} N+\left(1-b h_{z}^{2}\right) \sum S_{n}^{z} S_{n+1}^{z}-\frac{1}{2} \sum S_{n}^{x}
$$

Again, the original model (11) is equivalent to the effective model (40) in a sense, that the perturbation series for both models have the same order of divergencies (or power of $N$ ) at each order of $h_{z}$. But numerical factors at each order of $h_{z}$ can be different.

The advantage of this mapping lies in the fact that the effective model (40) is of the ITF type and, therefore, is exactly solvable one. The spectrum of $H_{\text {eff }}$ is

$$
\varepsilon_{k}^{2}=\frac{1+J^{2}}{4}+\frac{J}{2} \cos k
$$

with $J=1-b h_{z}^{2}$. So, according to the spectrum of the effective model (41) the magnetic field $h_{z}$ produces the gap in the spectrum

$$
m=\frac{b}{2} h_{z}^{2}
$$


As was noted above, apart from numerical factors the effective model (40) has the same form of perturbation series in $h_{z}$ as the original model (11). Therefore, we can check the form of the perturbation theory (30) by studying the perturbation series in $h_{z}$ of the exactly solvable model (40).

For large but finite systems the ground state energy (with $q=0$ ) and the first excited state (with $q=\pi$ ) of the Hamiltonian (40) are known exactly [19]:

$$
\begin{aligned}
& E_{0}=-a h_{z}^{2} N-\frac{1}{4} \sum_{j=1}^{N} \sqrt{g^{2}+4(1-g) \sin ^{2}\left(\frac{\pi j}{N}-\frac{\pi}{2 N}\right)} \\
& E_{\pi}=-a h_{z}^{2} N+\frac{g}{4}-\frac{1}{4} \sum_{j=1}^{N-1} \sqrt{g^{2}+4(1-g) \sin ^{2}\left(\frac{\pi j}{N}\right)}
\end{aligned}
$$

where $g=b h_{z}^{2}$.

The perturbation series for the ground state energy and the gap can be easily found by formal expansion of these expressions in small parameter $g$. This results in the scaling form of perturbation series for the mass gap:

$$
m=\frac{g}{4}+g^{2} N \sum_{n=0}^{\infty} d_{n}(g N)^{2 n}
$$

where constants $d_{n}$ determine the scaling function $G(x)$ of a scaling parameter $x=g N$

$$
m=\frac{g}{4}+g G(x)
$$

The scaling function $G(x)$ was found in [19]. In the thermodynamic limit $x \rightarrow \infty$ the function $G(x) \rightarrow 1 / 4$, resulting in the gap (42). One can see that the scaling parameter and the form of the perturbation series (44) coincides with Eq.(33).

Now we define the constants $a$ and $b$ in Eq.(40), so that the second order corrections to the ground state energy and the gap of the effective model (40) numerically coincide with Eqs. (27) and (35). This leads to the values

$$
\begin{aligned}
& a=0.190(1) \\
& b=0.750(1)
\end{aligned}
$$

The mass gap of the effective model (40) with constants $a$ and $b$ defined above is

$$
m=0.375(1) h_{z}^{2}
$$

Surprisingly, the value of the mass gap of the effective model turns out to be very close to one found numerically for the original model (Eq.(36)). It means, that the gap scaling function $G(x)$ of the effective model (40) has the same thermodynamic limit as that for the model (11). Moreover, we have calculated numerically the fourth order corrections in $h_{z}$ to the ground state energy and the gap for the models (11) and (40) and found perfect numerical agreement (the second order corrections in $h_{z}$ for both models coincide by the definition (46)). Therefore, we expect that the mapping of the model (11) at $h_{x}=1 / 2$ to the model (40) with constants $a$ and $b$ defined in (46) is exact for low-lying excitations in the thermodynamic limit.

The mapping of the model (11) to the effective ITF model can be extended to the case $h_{x} \neq 1 / 2$. For this case the effective Hamiltonian becomes

$$
H_{\mathrm{eff}}=-a\left(h_{x}\right) h_{z}^{2} N+\left(1-b\left(h_{x}\right) h_{z}^{2}\right) \sum S_{n}^{z} S_{n+1}^{z}-h_{x} \sum S_{n}^{x}
$$

where now the constants $a, b$ are the functions of $h_{x}$. The functions $a\left(h_{x}\right)$ and $b\left(h_{x}\right)$ are defined in such a way that the second order corrections to the ground state energy and the gap for the effective model (40) coincide with those for the original model (11).

Using the effective Hamiltonian (48) one can calculate the susceptibility $\chi_{z}\left(h_{x}\right)$. In the vicinity of the critical point $h_{x}=1 / 2$ it has a logarithmic singularity

$$
\chi_{z}\left(h_{x}\right)=\chi_{z c}-\frac{b}{\pi}\left(h_{x}-\frac{1}{2}\right) \log \left(h_{x}-\frac{1}{2}\right)
$$

with $\chi_{z c}=0.1412$ from Eq.(27) and $b=0.75$ (Eq.(46) ). 


\section{THE MULTICRITICAL POINT}

Another exactly solvable limit of the model (2) is the case $h_{x}=0$. The model at $h_{x}=0$ is the classical one. At $h_{z}<1$ its ground state is the antiferromagnet. At $h_{z}=1$ the first order phase transition to the ferromagnetic ground state occurs. This is so-called multicritical point. The Hamiltonian (2) at $h_{z}=1$ has a form

$$
\begin{aligned}
H & =H_{0}+V \\
H_{0} & =-\frac{N}{4}+\sum\left(S_{n}^{z}-\frac{1}{2}\right)\left(S_{n+1}^{z}-\frac{1}{2}\right) \\
V & =-\frac{h_{x}}{2}\left(S^{+}+S^{-}\right)
\end{aligned}
$$

where $S^{ \pm}=\sum S_{n}^{ \pm}$.

The ground state of $H_{0}$ is macroscopic degenerate: all spin configurations, excluding those with two neighbor spins pointing down, have the same energy $-\frac{N}{4}$. The number of these states is $\left(\frac{1+\sqrt{5}}{2}\right)^{N}[20]$. The transverse field $h_{x}$ lifts the degeneracy. The exact calculation of the first order correction in $h_{x}$ for $N \gg 1$ is rather complicated because it involves the exponentially large number of degenerate states. We carried out the approximate calculation of the perturbation theory in $V$ within degenerate manifold using a simple variational function in the form

$$
\Psi=\sum_{m=0}^{N / 2} c_{m} \Psi_{m}
$$

where $\Psi_{m}$ are the sum (with equal weights) of all admissible states with $m$ spins down:

$$
\Psi_{m}=w_{m}^{-\frac{1}{2}}\left(S^{-} P\right)^{m} \Psi_{F}
$$

Here $P$ is a projector excluding states with two neighbor down spins, $\Psi_{F}$ is the ferromagnetic state with all spins up and the normalization factors $w_{m}$ are

$$
w_{m}=\frac{(N-m-1) ! N}{m !(N-2 m) !}
$$

Matrix elements of $V$ with respect to $\Psi_{m}$ are

$$
\left\langle\Psi_{m} V \Psi_{m^{\prime}}\right\rangle=\frac{h_{x}}{2}\left(s_{m} \delta_{m^{\prime}, m-1}+s_{m+1} \delta_{m^{\prime}, m+1}\right)
$$

where

$$
s_{m}=\sqrt{\frac{m(N-2 m+2)(N-2 m+1)}{N-m}}
$$

Coefficients $c_{m}$ in (51) obey the equations

$$
\left(E+\frac{N}{4}\right) c_{m}=\frac{h_{x}}{2}\left(s_{m+1} c_{m+1}+s_{m} c_{m-1}\right)
$$

The quantity $s_{m}$ has a sharp maximum for $N \gg 1$ at $m_{0}=\frac{3-\sqrt{5}}{4} N$ and $s_{m_{0}}$ is

$$
s_{m_{0}}=\frac{N}{2}(\sqrt{5}-1) \sqrt{\sqrt{5}-2}
$$

The ground state energy in the thermodynamic limit is defined as a lowest eigenvalue of Eqs.(56), which is

$$
E_{0}=-\frac{N}{4}-h_{x} s_{m_{0}}=-\frac{N}{4}-0.30028 N h_{x}
$$

In the frame of variational function approach (51) the ground state magnetizations $\left\langle S_{n}^{z}\right\rangle$ and $\left\langle S_{n}^{x}\right\rangle$ are

$$
\begin{aligned}
\left\langle S_{n}^{z}\right\rangle & =\frac{1}{2}-\frac{m_{0}}{2 N}=0.309 \\
\left\langle S_{n}^{x}\right\rangle & =\frac{s_{m_{0}}}{N}=0.300028
\end{aligned}
$$


The function (51) has a momentum $q=0$. To calculate the spectrum in the first order in $h_{x}$ it is necessary to choose the variational function of the type (51) with momentum $q$. We omit here rather cumbersome calculations, which shows that the mass gap corresponds to $q=\pi$ and

$$
m=h_{x}
$$

The numerical diagonalization of finite cyclic systems shows very rapid exponential convergence to the thermodynamic limit. The extrapolated values for the ground state energy and the mass gap (at $q=\pi)$ for $h_{x} \ll 1$ are

$$
\begin{aligned}
\frac{E_{0}}{N} & =-\frac{1}{4}-0.3017(1) h_{x} \\
m & =0.4841(1) h_{x}
\end{aligned}
$$

Comparison of these results with Eq.(58) shows that the variational ground state energy differs from the 'exact' one within $0.4 \%$. As follows from Eq.(61) the mass gap in the multicritical point opens linearly with $h_{x}$, which is correctly described by the variational approach (60).

\section{THE CRITICAL LINE}

In general, the critical line at $h_{x} \neq 0$ and $h_{z} \neq 0$ can not be found exactly. To obtain it we used the DMRG technique 14. We have performed DMRG calculations using both the infinite-size and the finite-size DMRG algorithms. We calculated the ground state energy $E_{0}(N)$ and two lowest excitations $m_{1}(N)=E_{1}(N)-E_{0}(N)$ and $m_{2}(N)=$ $E_{2}(N)-E_{0}(N)$.

In order to check the accuracy of the DMRG method we compared the obtained results with the exact ones for the ITF model. We used the infinite-size algorithm and open boundary conditions. The dependence of the results on the number of retained states $s$ in the DMRG computation and on a number $N_{R G}$ of DMRG steps $\left(N=2 N_{R G}+2\right)$ has been investigated. We have found that the calculation with $s=25$ gives satisfactory accuracy up to $N=300$. For example, relative errors in the ground state energy and in the mass gap at $h_{x}=\frac{1}{2}$ are $\frac{\Delta E_{0}}{E_{0}}=10^{-9}\left(10^{-7}\right)$ and $\frac{\Delta m}{m}=10^{-7}\left(10^{-5}\right)$ for $N=100(300)$. The accuracy becomes better when the value $\left|h_{x}-\frac{1}{2}\right|$ is increased.

The critical field $h_{x c}\left(h_{z}\right)$ at a fixed value $h_{z}\left(0<h_{z}<1\right)$ is determined by vanishing of the gaps $m_{1}$ and $m_{2}$. Below the critical field the mass gap $m_{1}(N) \rightarrow 0$ exponentially with $N$. This behavior confirms the fact that the ground state is doubly degenerate in the thermodynamic limit at $h_{x}<h_{x c}\left(h_{z}\right)$. The true mass gap in this region is defined by the value of $m_{2}$. The typical behavior of the gaps $m_{1}$ and $m_{2}$ extrapolated to the thermodynamic limit is shown on Fig. 3. We note that at $h_{x}>h_{x c}\left(h_{z}\right)$ the gaps $m_{1}$ and $m_{2}$ coincide at $N \rightarrow \infty$.

The critical line obtained from the DMRG results extrapolated to the thermodynamic limit is shown on Fig. 11 In the plane $\left(h_{x}, h_{z}\right)$ it connects two limiting critical points studied in previous sections: the critical point $\left(h_{x}=\frac{1}{2}, h_{z}=0\right)$ of the ITF model and the multicritical point $\left(h_{x}=0, h_{z}=1\right)$. Now we discuss the properties of the model (2) near the critical line.

The ITF model in the critical point is described by a conformal field theory with a central charge $c=\frac{1}{2}$. We expect that the ITF model is generic for the model (2) on the whole critical line (except the multicritical point $\left.h_{x}=0, h_{z}=1\right)$. To verify this suggestion we estimated the value of $c$ on the critical line. For this we used well known fact 21] that for conformal invariant model with periodic boundary conditions the central charge appears at $1 / N$ correction to the ground state energy

$$
E_{0}=e_{\infty} N-\frac{\pi c v}{6 N}
$$

where $e_{\infty}$ is the ground state energy per site in the thermodynamic limit and $v$ is the sound velocity.

At first, we calculated the sound velocity $v$ as (similar to the spectrum of the ITF model (18) the sound velocity on the whole critical line is determined at $k=\pi$ )

$$
v(N)=\frac{N}{2 \pi}\left[E\left(\pi-\frac{2 \pi}{N}\right)-E(\pi)\right]
$$

We carried out these calculations using the numerical diagonalization of the Hamiltonian (2) with the periodic boundary condition for $N \leq 14$. The size extrapolation is carried out by formula $v(N)=v+a N^{-2}$. The example of the extrapolation procedure is shown on Fig. [4 The dependence of $v$ on $h_{z}$ along the critical line is shown on Fig. [5] 


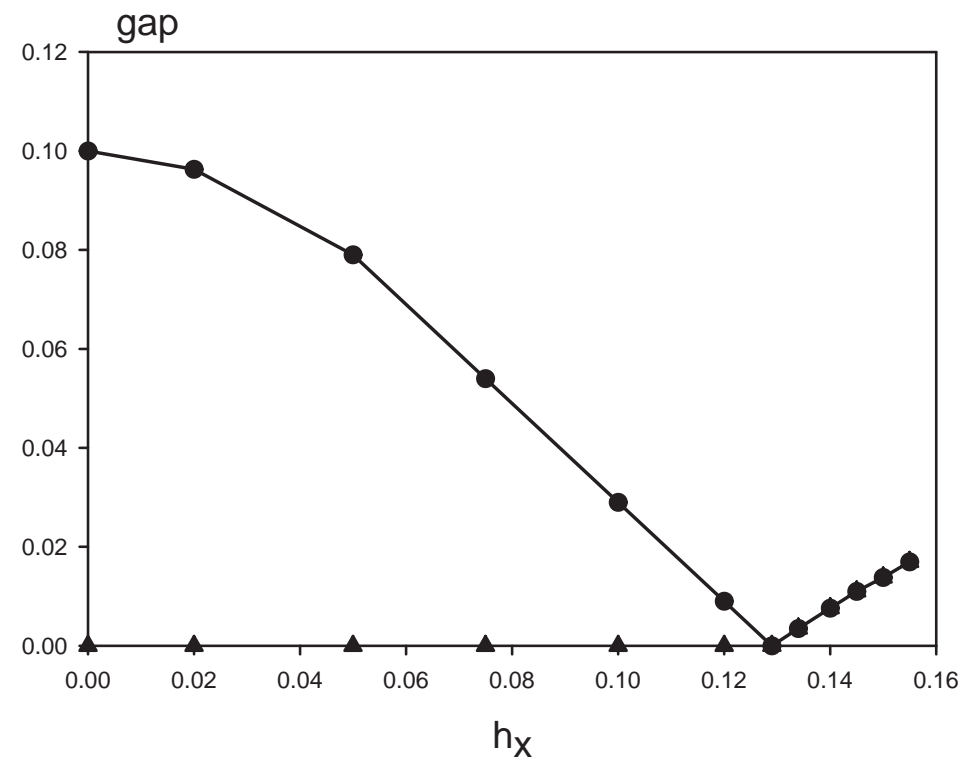

FIG. 3: The mass gaps $m_{1}$ (triangles) and $m_{2}$ (circles) as functions of the transverse field $h_{x}$ at $h_{z}=0.9$ from the DMRG calculations extrapolated to the thermodynamic limit.

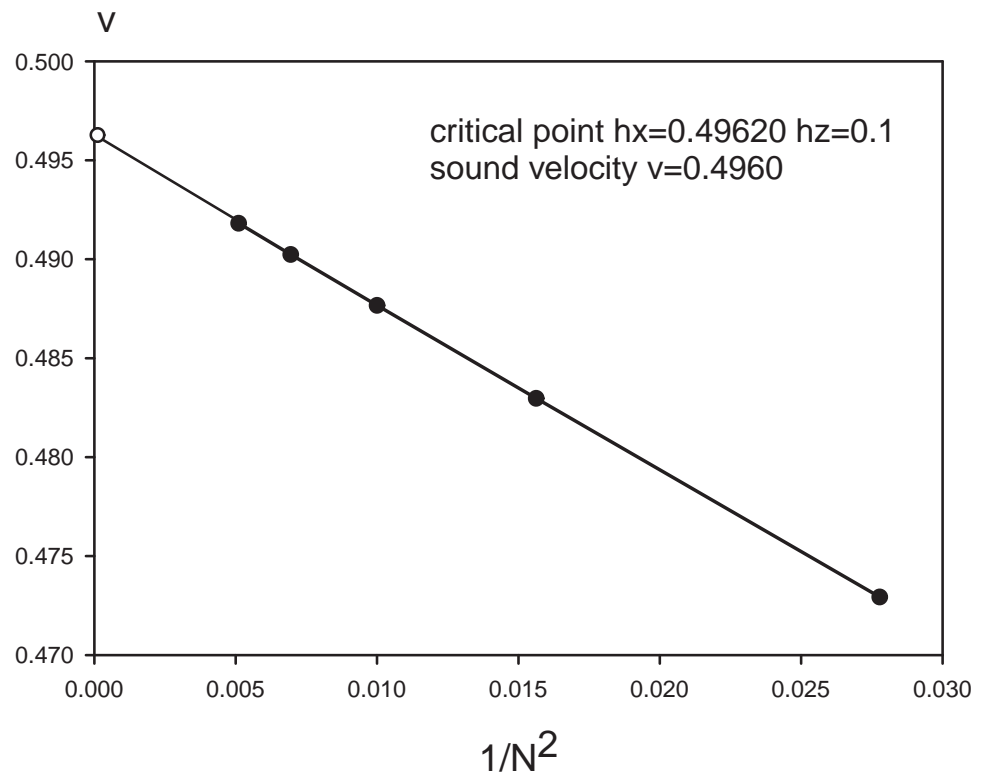

FIG. 4: The extrapolation procedure of finite size dependence of the sound velocity in the critical point $h_{x}=0.4962, h_{z}=0.1$. $(v=0.4960)$.

After that, the central charge $c$ has been calculated using Eq. (62). The size extrapolation shows that the central charge is $c=0.500(1)$ for all calculated critical points.

The critical exponents $\nu$ of the ground state correlation function $\left\langle\widehat{O}_{1} \widehat{O}_{R}\right\rangle \sim R^{-\nu}(R \gg 1)$ is defined by the scaling dimension $X$ of the operator $\widehat{O}$ and $\nu=2 X[22]$. The scaling dimension is related to the finite-size correction of the lowest excited eigenstate which can be reached from the ground state by the operator $\widehat{O}$. The finite-size correction to this excitation energy is

$$
\Delta E_{i}=E_{i}-E_{0}=\frac{2 \pi v}{N} X_{i}+o\left(\frac{1}{N}\right)
$$

For the ITF model at $h_{x}=\frac{1}{2}$ the sound velocity is $v=\frac{1}{2}$. The ground state has a momentum $q=0$ and the lowest 


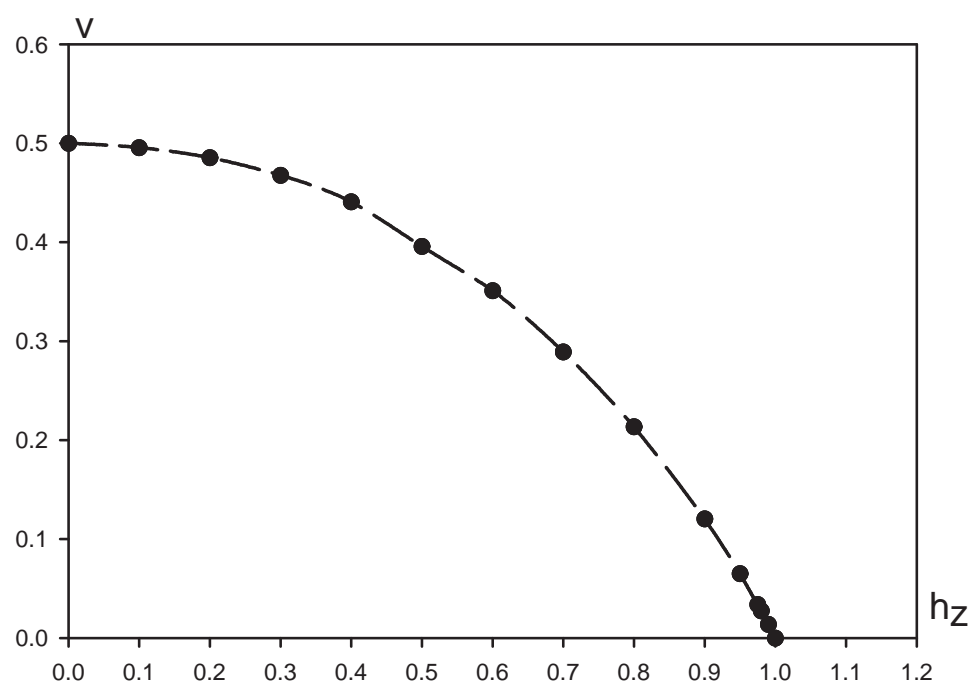

FIG. 5: The dependence of the sound velocity on $h_{z}$ along the critical line.

excitation energy in the sector with momentum $q=0$ is

$$
E(q=0)-E_{0}=2 \sin \frac{\pi}{2 N}
$$

and in the sector with $q=\pi$ is

$$
E(q=\pi)-E_{0}=\frac{1}{2} \tan \frac{\pi}{4 N}
$$

Thus, the scaling dimensions are $X_{0}=1$ and $X_{\pi}=\frac{1}{8}$. The corresponding associated operators are

$$
\begin{aligned}
& \widehat{O}_{q=0}=\sum_{n} S_{n}^{x}, \\
& \widehat{O}_{q=\pi}=\sum_{n}(-1)^{n} S_{n}^{z}
\end{aligned}
$$

which is in accord with the well known results [17] for the asymptotic of the correlation functions

$$
\begin{aligned}
\left\langle S_{0}^{x} S_{R}^{x}\right\rangle-\left\langle S_{0}^{x}\right\rangle^{2} & \sim R^{-2} \\
\left\langle S_{0}^{z} S_{R}^{z}\right\rangle & \sim \frac{(-1)^{R}}{R^{1 / 4}}
\end{aligned}
$$

Using Eq.(64) we numerically checked that the scaling dimensions related to the finite-size corrections of the lowest excited eigenstates on the critical line remain as in ITF model $X_{0}=1$ and $X_{\pi}=\frac{1}{8}$. Therefore, we conclude that the model (2) on the critical line belongs to the universality class of the ITF model. This means that in accord with the prediction of the classical approach to the left of the critical line the staggered magnetizations $\left\langle(-1)^{n} S_{n}^{z}\right\rangle$ and $\left\langle(-1)^{n} S_{n}^{x}\right\rangle$ exist. But in contrast to the classical approach they vanish on the critical line with the critical exponent $\frac{1}{8}$. The mass gap is closed on the critical line and the critical exponent for the gap is equal to unity, i.e. the scaling behavior of the gap near the critical line is linear. For the particular case $h_{z}=0.5$, this is illustrated on Fig. 6 where the scaling plot for the scaled mass $N m_{1}$ with $N\left(h_{x}-h_{x c}\right)$ is shown.

The behavior of the critical line near the ITF point $h_{x}=1 / 2, h_{z}=0$ can be found from the following consideration. As it was established above in the vicinity of the critical line the gap is proportional to deviation from the line. This is valid for any direction of deviation except the direction at a tangent to the critical line. In the vicinity of the ITF critical point for fixed $h_{z} \ll 1$ according to Eq. (17) the gap is $m=\left|h_{x}-h_{x c}\left(h_{z}\right)\right|$. On the other hand, the gap is given by Eq.(36). Combining these two expressions for the gap on the line $h_{x}=1 / 2$, we obtain equation for the critical line in the vicinity of the point $h_{x}=1 / 2, h_{z}=0$ as

$$
h_{x \mathrm{c}}\left(h_{z}\right)=\frac{1}{2}-0.37 h_{z}^{2}
$$




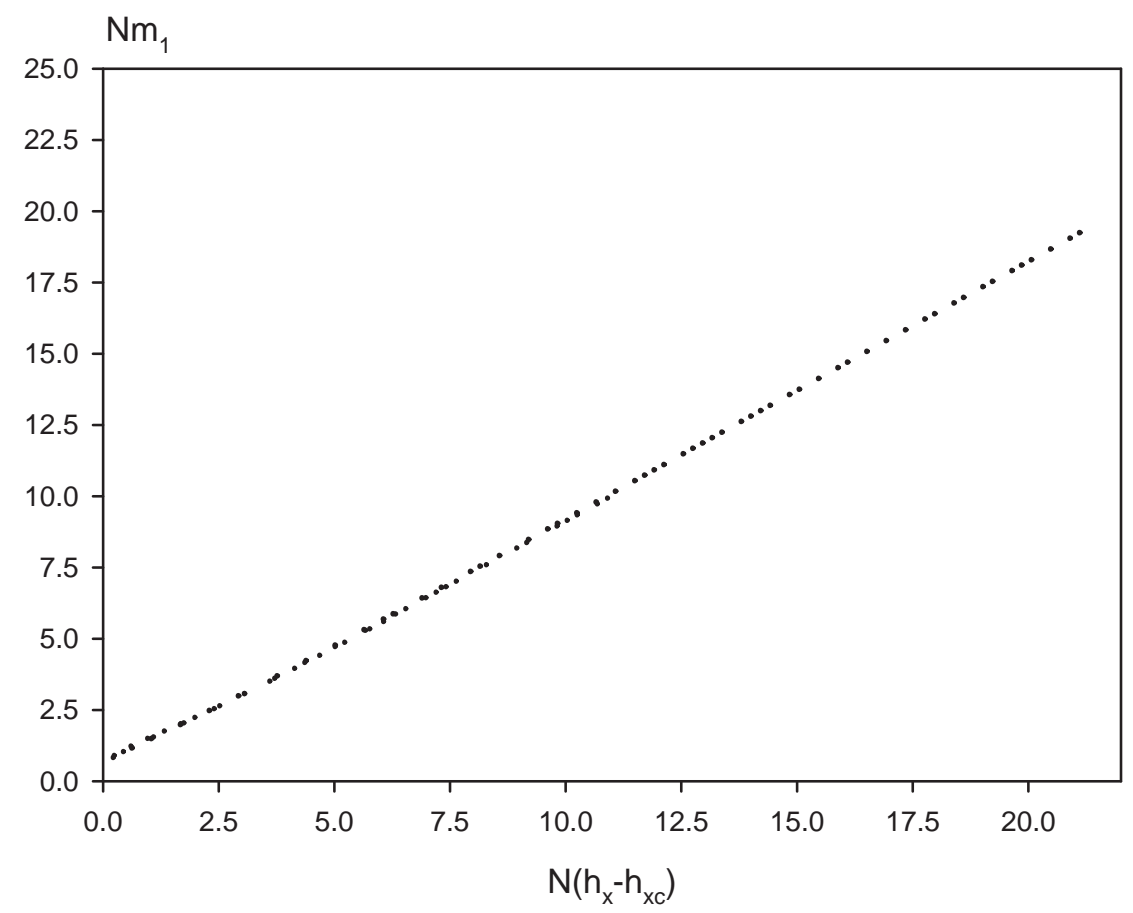

FIG. 6: Scaled mass gap $N m_{1}$ for $h_{z}=0.5$ with various values of $N$ plotted as a function of the scaled field $N h_{x}$.

This expression for the critical line is in a very good agreement with our numerical estimations up to $h_{z} \sim 0.5$.

In the vicinity of the multicritical point the form of the critical line can be found in a similar way. At $h_{z}=1$ and $h_{x} \ll 1$ the mass gap is proportional to $h_{x}$ (see Eq.(61)). On the other hand, for fixed $h_{x} \ll 1$ this gap is proportional to a deviation from the critical line $m \sim\left(h_{z}-h_{z c}\right)$. Therefore, the critical line near the multicritical point behaves as $h_{x c}=A\left(1-h_{z}\right)$. The numerical coefficient $A$ has been found from the DMRG calculation of the critical line in the vicinity of the multicritical point. As a result, the form of the critical line at $h_{x} \ll 1$ is

$$
h_{x \mathrm{c}}\left(h_{z}\right)=1.50\left(1-h_{z}\right)+O\left(\left(1-h_{z}\right)^{2}\right)
$$

This form differs from that given in [10], where $h_{x c}\left(h_{z}\right) \sim\left(1-h_{z}\right)^{0.75}$.

\section{RELATION WITH THE TWO-DIMENSIONAL ISING MODEL}

It is well-known that the critical properties of the two-dimensional Ising model are equivalent to those of the onedimensional ITF model [23]. Using the formalism of Ref. 23] one can show that the quantum Hamiltonian (22) is related to the transfer matrix of the strong anisotropic (quasi-one-dimensional) Ising model in the uniform magnetic field. This model describes ferromagnetic Ising chains weakly antiferromagnetically coupled with each other. The Hamiltonian of the model is

$$
H=-J_{1} \sum \sigma_{n, m} \sigma_{n+1, m}+J_{2} \sum \sigma_{n, m} \sigma_{n, m+1}-h \sum \sigma_{n, m}
$$

where $\sigma_{n, m}= \pm 1, J_{1} \gg J_{2}>0$.

The relation between the model (2) and the model (71) are given by

$$
\begin{aligned}
2 h_{x} \beta J_{2} & =e^{-2 \beta J_{1}} \ll 1 \\
h & =2 h_{z} J_{2}
\end{aligned}
$$

with $\beta=1 / k T$.

The equivalence of these two models means that the model (71) undergoes the phase transition from the ordered (at $T<T_{c}$ ) to the disordered (at $T>T_{c}$ ) phase. The critical line $T_{c}(h)$ of the quasi-one dimensional model are related 
to the critical line $h_{x \mathrm{c}}\left(h_{z}\right)$ of the model (2). By use of mapping (72) we obtain that the critical temperature $T_{c}(h)$ is

$$
k T_{c}(h)=\frac{2 J_{1}}{\log \left(\frac{J_{1}}{J_{2} h_{x}\left(h / 2 J_{2}\right)}\right)}
$$

In particular, at $h \rightarrow 0$

$$
k T_{c}=\frac{2 J_{1}}{\log \left(\frac{2 J_{1}}{J_{2}}\right)}
$$

and $T_{c} \rightarrow 0$ when $h \rightarrow 2 J_{2}$ as

$$
k T_{c}=\frac{2 J_{1}}{\log \left(\frac{J_{1}}{2 J_{2}-h}\right)}
$$

The order parameter $\frac{1}{2}\left\langle\sigma_{n, m}+\sigma_{n, m+1}\right\rangle$ vanishes at $T \rightarrow T_{c}$ with the critical exponent $1 / 8$. We note that the phase transition does not occur if $J_{2}<0$ as well as in the ferromagnetic version of the model (2).

The free energy of the model (71) is related to the ground state energy of the Hamiltonian (2) by

$$
F=4 J_{2} E_{0}\left(h_{x}, h_{z}\right)
$$

According to Eqs.(49), (72) and (76) the zero-field susceptibility in the vicinity of the critical temperature $T_{c}(0)$ can be obtained using exactly solvable effective model (40). At $\left|T-T_{c}(0)\right| \ll T_{c}(0)$ the susceptibility is

$$
\chi J_{2}=0.1412-0.24 \frac{J_{1}}{k T_{c}(0)} \frac{T-T_{c}(0)}{T_{c}(0)} \log \left(\frac{T-T_{c}(0)}{T_{c}(0)}\right)
$$

The last equation has a form coinciding with the results obtained for the two-dimensional antiferromagnetic Ising model by the series expansion method [24].

\section{SUMMARY}

We have studied the antiferromagnetic Ising chain in the mixed transverse and longitudinal magnetic field. It was shown that the quantum phase transition existing in the ITF model remains in the presence of the uniform longitudinal field. Using the DMRG simulations we have found the critical line in the $\left(h_{x}, h_{z}\right)$ plane where the mass gap is closed and the staggered magnetizations along the $X$ and $Z$ axes vanish. It is found numerically that the model on the critical line is described by the conformal field theory with the central charge $c=1 / 2$, i.e. it belongs to the universality class of the ITF model.

The scaling behavior in the vicinity of the ITF critical point is studied in detail. It is shown that the mass gap is proportional to $h_{z}^{2}$ and the contributions to it are given not only by the second order correction but also by all other divergent orders of the perturbation series in $h_{z}$. Besides, the analysis of the perturbation theory shows that the considered model at $h_{z} \ll 1$ can be mapped to the effective ITF model with renormalized parameters depending on $h_{z}$. In a framework of the effective ITF model the behavior of the susceptibility at $h_{x} \sim 1 / 2$ is determined.

The behavior of the model in the vicinity of the multicritical point is investigated. Using both the variational approach and the numerical diagonalization results we have found that the mass gap is proportional to $h_{x}$. Close to the multicritical point the form of the critical line is linear.

Of course, the considered model is the simplest case of the $X X Z$ model (1). It is interesting to extend the present analysis to this model as well as to study effects of interchain interactions in quasi-one-dimensional generalization of the model (2).

The mapping of the quantum model (2) to the strongly anisotropic statistical two-dimensional Ising model in the uniform magnetic field was considered. The behavior of the susceptibility of this model near the critical temperature is found. We expect also, that the main features of the considered model (2) are valid for the statistical two-dimensional antiferromagnetic Ising model in the uniform magnetic field $h$. That is, the applied magnetic field does not smear the phase transition existing in the two-dimensional Ising model, which is generic case for the whole transition line $T_{c}(h)$ in the plane $(T, h)$. In particular, we believe that the analysis of the perturbation series in $h \ll 1$ in the same manner 
as was done in Sec.III gives the scaling behavior for the correlation length and the form of the critical line near the point $T_{c}(0)$ is $T_{c}(0)-T_{c}(h) \sim h^{2}$.

This work is supported under RFBR Grant No 03-03-32141 and ISTC No 2207.

* Electronic address: dmitriev@deom.chph.ras.ru

[1] R.Helfrich, M.Koppen, M.Lang, F.Steglich and A.Ochiku, J.Magn.Magn.Mat. 177, 309 (1998).

[2] I.Affleck and M.Oshikawa, Phys.Rev.B 60, 1038 (1999).

[3] G.Uimin, Y.Kudasov, P.Fulde and A.A.Ovchinnikov, Eur.Phys.J.B 16, 241 (2000).

[4] M.Kohgi et al., Phys.Rev.Lett. 86, 2439 (2001).

[5] S.Mori, J.-J.Kim and I.Harada, J.Phys.Soc.Jpn 64, 3409 (1995).

[6] Y.Hieida, K.Okunishi and Y.Akutsu, Phys.Rev.B 64, 224422 (2001).

[7] D.V.Dmitriev, V.Ya.Krivnov, A.A.Ovchinnikov and A.Langari, Zh. Eksp. Teor. Fiz. 122, 624 (2002) [JETP 95, 538 (2002)]; D.V.Dmitriev, V.Ya.Krivnov and A.A.Ovchinnikov, Phys. Rev. B 65, 172409 (2002).

[8] A.Dutta, D.Sen, Phys. Rev. B 67, 094435 (2002).

[9] M.Kenzelmann et. al., Phys. Rev. B 65, 144432 (2002).

[10] P.Sen, Phys. Rev. E 63, 16112 (2001).

[11] H.C.Fogedby, J. Phys. C: Solid State Phys., Vol. 11, 2801 (1978).

[12] A.B.Zamolodchikov, Int. J. Mod. Phys. A4, 4235 (1989).

[13] V.A.Fateev, Phys. Lett. B 324, 4551 (1994).

[14] S.R.White, Phys. Rev. B 48, 10345 (1993).

[15] P.Pfeuty, Ann. of Phys. 57, 79 (1970).

[16] E.Lieb, T.Schultz, D.Mattis, Ann. Phys. (N.Y.) 16, 407 (1961).

[17] E.Barouch and B.M.McCoy, Phys. Rev. A 3, 786 (1971).

[18] G.Muller and R.E.Shrock, Phys.Rev.B 30, 5254 (1984).

[19] C.J.Hamer and M.N.Barber, J. Phys. A: Math. Gen. 14, 241 (1981).

[20] C.Domb, Adv.Phys. 9, 149 (1960).

[21] J.L.Cardy in Phase transition and critical phenomena, eds. Domb and Lebowitz, Vol.XI, (Academic, New York, 1986).

[22] A.O.Gogolin, A.A.Nersesyan, A.M.Tsvelik, Bosonization and Strongly Correlated Systems (Cambridge University Press, Cambridge, 1998).

[23] E.Fradkin and L.Susskind, Phys. Rev. D17, 2637 (1978); J.B.Kogut, Rev.Mod.Phys. 51, 659 (1979).

[24] M.E.Sykes and M.Fisher, Physica 28, 919 (1962); M.E.Sykes, D.S.Gaunt, P.D.Robert and J.A.Wyles, J.Phys. A5, 667 (1972). 